استخدام مفاهيم الاتزان والايناميكا الحرارية لامتزاز النحاس في بعض الترب الكلسية

محمد مالك ياسين و سلوى جمعة فاخر

قسم علوم التربة و الموارد المائية /كلية الزر اعة /جامعة البصرة -البصرة-العر اق

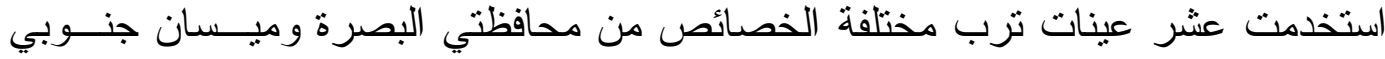

العر اق لدر اسة ميكانيكية امتز از النحاس. حيث أضيف ايونات النحاس بالمـستويات $0.01 \mathrm{M} \mathrm{NaCl} 0.01 \mathrm{M} \mathrm{CaCl}_{2}$ و 16, 32 mg Cu kg $\mathrm{mg}^{-1}$ soil)

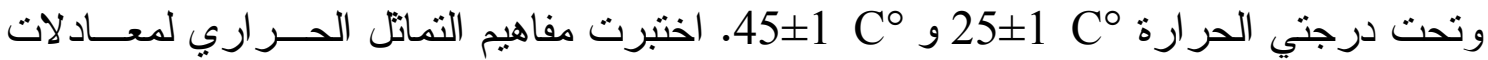

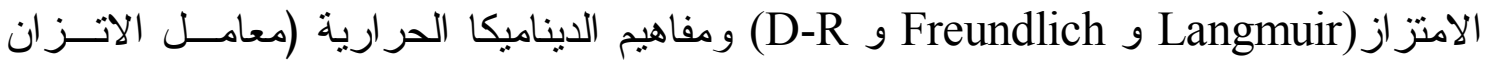

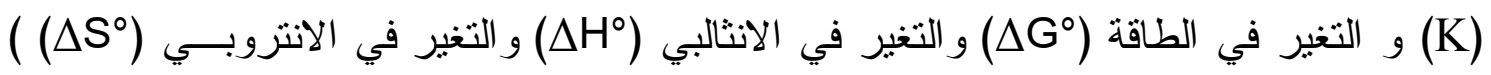

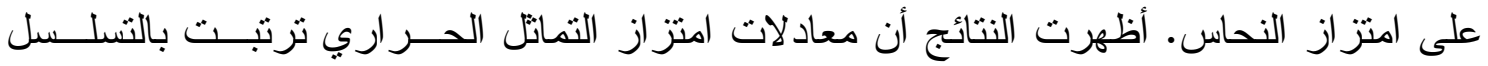

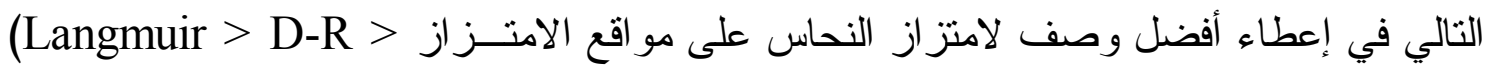

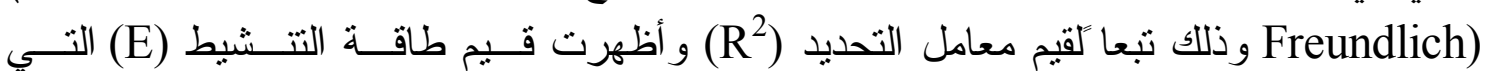

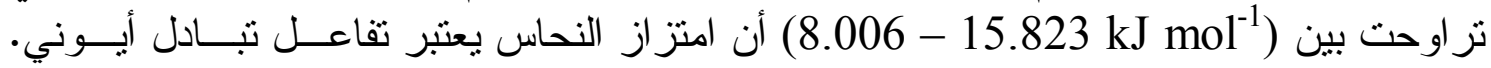
و أثنارت قيم $\Delta \mathrm{G}^{\circ}$ لإمتز از النحاس في التزب المدروسة الى أن تفاعل الامتز از للنحاس تلقـائي

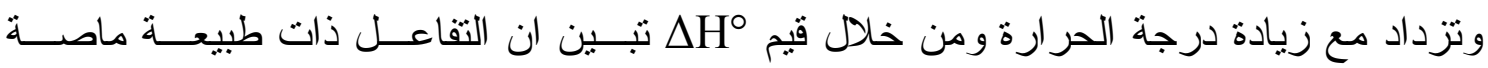

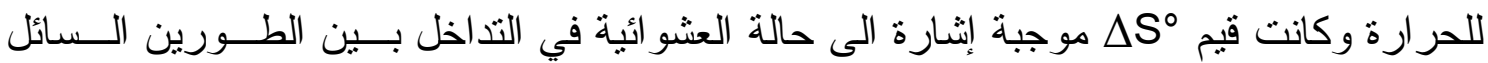

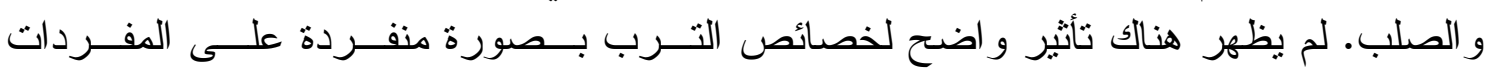

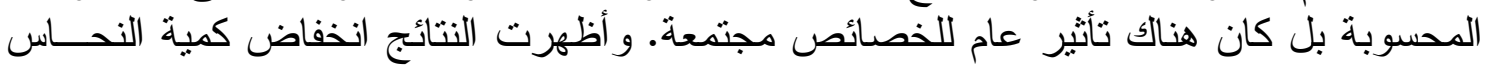

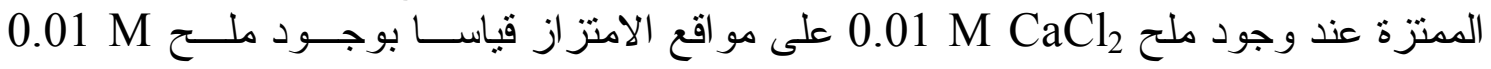
. $\mathrm{NaCl}$ الكلمات الدفتاحية: الامتز/ز - الاتزان - الديناميكا الحرارية - النحاس.

المقدمة

يدخل النحاس النظام الزر اعي من الاضافات للمبيدات الفطرية ومن مخلفات المصانع منل

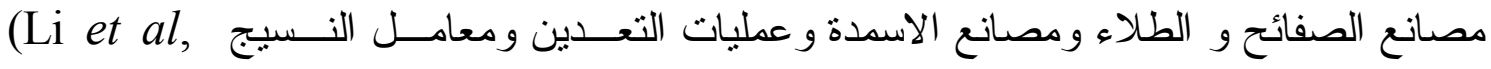
(لتر 2007; Srivastava et al, 2006)

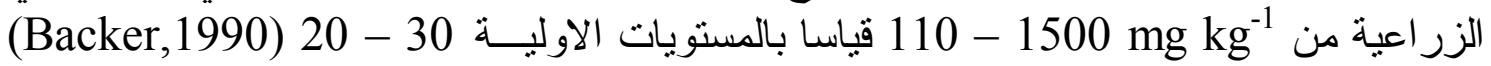
وتعتبر حالة نقص النحاس في الترب الكلسية حالة شائعة إذ من المعتقد ان ذلك نتيجة حدوث تفاعلات بين النحاس الذائب و الطور الصلب في الترب مما يؤدي الى انخفاض جاهزيته

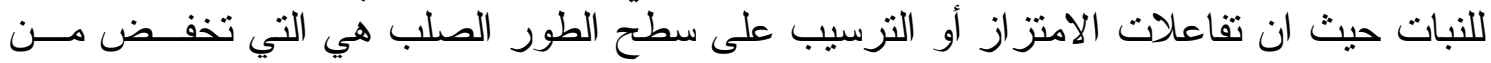

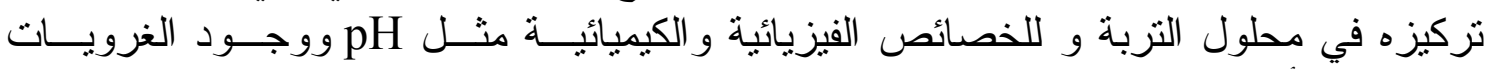

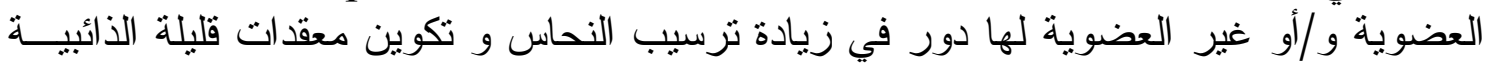
(Lopez et al ,1998)

يوجد ثلاثة انواع من الامتز از هي الفيزيائي و الكيميائي و التبــادلي (Sawyer and Mc )

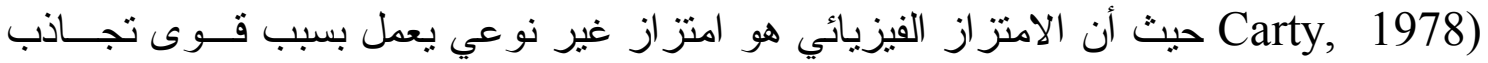

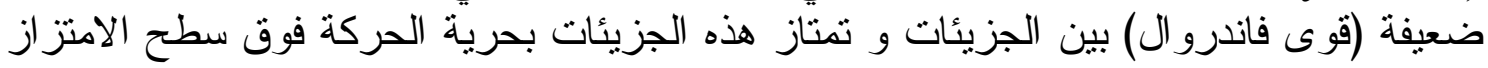


وقد تتكثف هذه الجزيئات بشكل طبقات على سطح الامتز از ويكون الامتز از الفيزيــائي عكـسياً

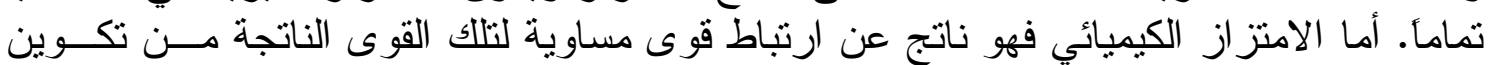

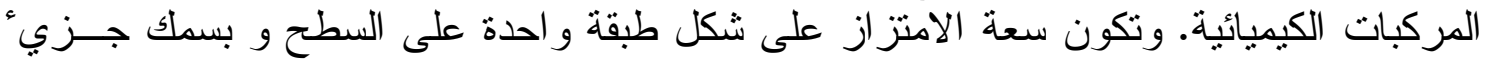

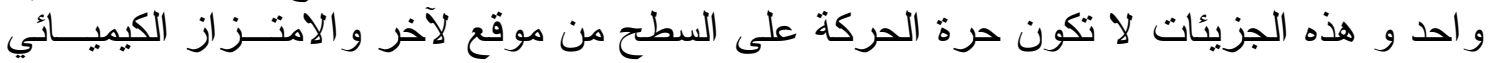
نادر آ ما يكون عكسي. (Chen et al, 2001). تعتبر نماذج التماتلك الحراري Isotherm models مثل معادلات فرنـــليش

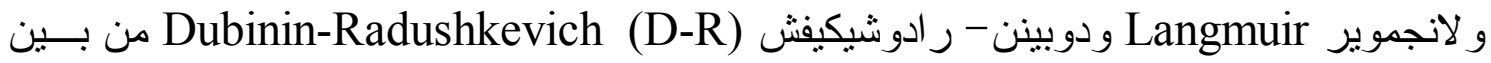

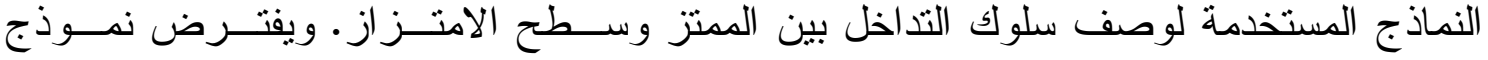

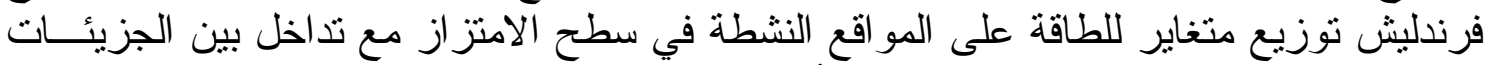

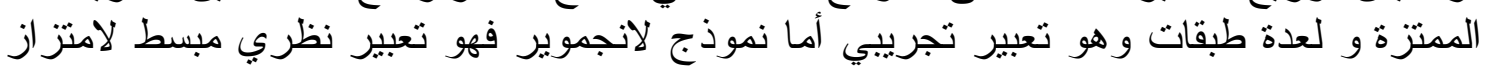

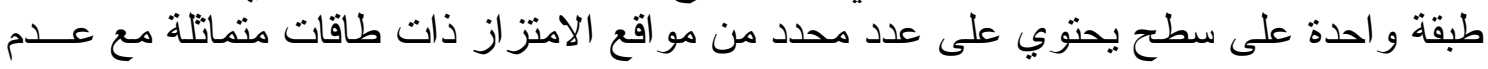

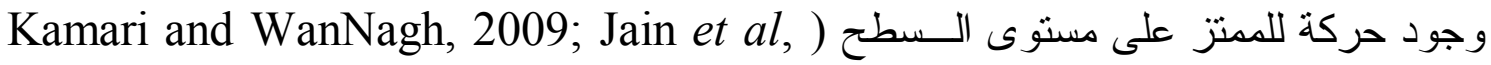

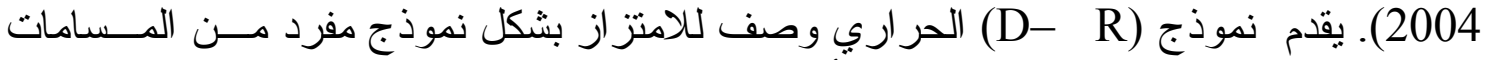
المتماثلة، وهو لا يفترض سطح متجانس أو جهد امتصاص ثنابت حيث يعطي معنى للجهد مــن

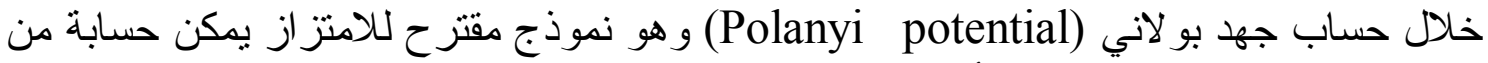
خلال الاتزان للجهد الكيميائي للأيون قرب السطح و جهدة الكيميائي عند مسافة معينة بعيدة عن من الان السطح (2003 (Kilislioglu and Bilginn).

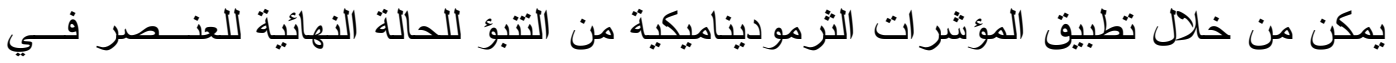

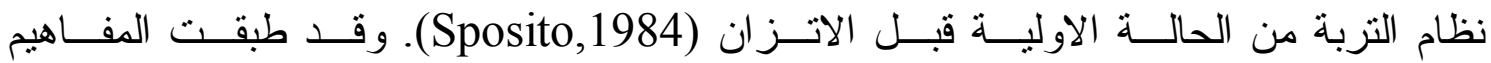

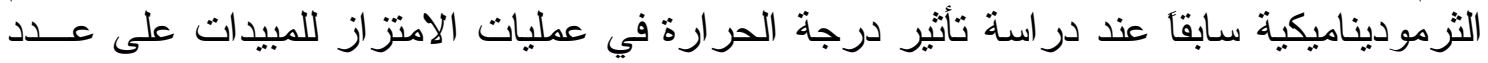
من مو اد الامتز از (Mills and Bigger, 1969 a,b,c).

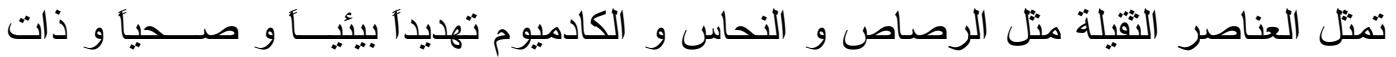

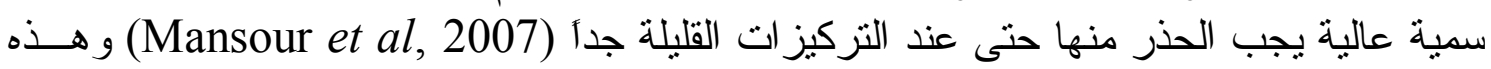
العناصر لا تتحل في البيئة وهي مؤذية لأنو اع مختلفة من الاحياء ولهذا فأن أز التها مـــن البيئـــة

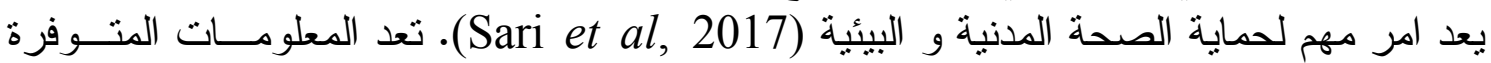

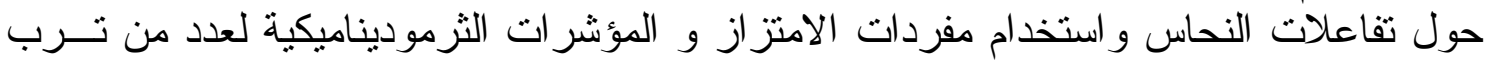

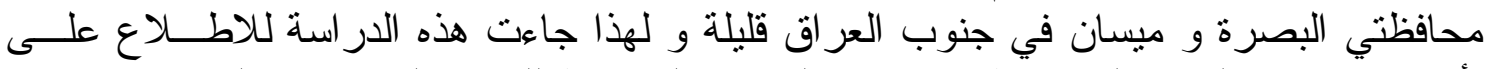

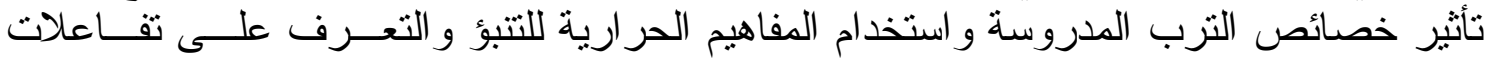
عنصر النحاس. المو اد و طر ائق العمل

جمعت عينات التزب قيد الدر اسة من عشرة مناطق موزعة في محافظتي البصرة وميسان

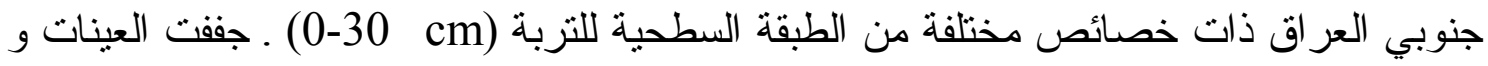

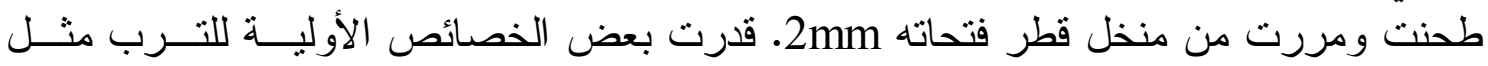

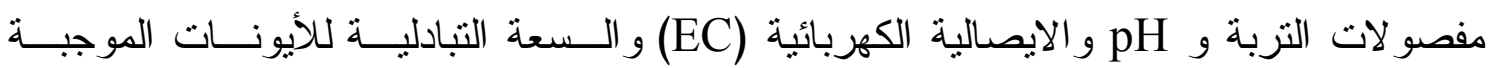
(CEC)

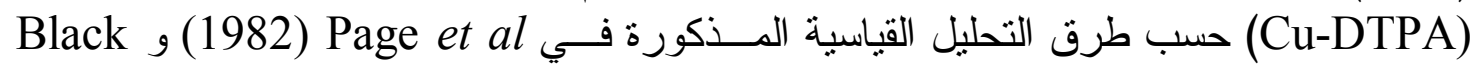
(1965) وكما موضت في جدول (1). 
جدول 1. بعض الخصائص الأولية للترب تحت الاراسة

\begin{tabular}{|c|c|c|c|c|c|c|c|c|c|}
\hline $\begin{array}{c}\text { Cu- } \\
\text { DTPA }\end{array}$ & $\mathrm{CaCO}_{3}$ & Sand & Silt & Clay & O.M & EC & CEC & \multirow[t]{2}{*}{$\begin{array}{l}\mathrm{pH} \\
1: 1\end{array}$} & \multirow{2}{*}{ تلترب } \\
\hline $\mathrm{mg} \mathrm{Kg}^{-1}$ & $\mathrm{~g} \mathrm{Kg}^{-1}$ & $\mathrm{~g} \mathrm{Kg}^{-1}$ & $\mathrm{~g} \mathrm{Kg}^{-1}$ & $\mathrm{~g} \mathrm{Kg}^{-1}$ & $\mathrm{~g} \mathrm{Kg}^{-1}$ & dS. $m^{-1}$ & $\mathrm{cmol}_{+} \mathrm{Kg}^{-1}$ & & \\
\hline 16.60 & 302.30 & 59.98 & 530.00 & 410.02 & 9.24 & 7.50 & 38.50 & 8.30 & 1 \\
\hline 20.40 & 321.30 & 72.90 & 460.40 & 466.70 & 20.00 & 7.00 & 13.60 & 8.30 & 2 \\
\hline 14.50 & 283.60 & 85.70 & 453.70 & 460.60 & 8.48 & 9.20 & 14.20 & 8.00 & 3 \\
\hline 14.20 & 405.00 & 74.70 & 452.90 & 472.40 & 11.60 & 9.10 & 16.00 & 7.95 & 4 \\
\hline 17.50 & 364.70 & 59.98 & 520.00 & 420.02 & 5.92 & 7.50 & 18.60 & 8.50 & 5 \\
\hline 5.40 & 200.90 & 898.10 & 39.30 & 62.60 & 4.68 & 2.60 & 4.70 & 8.19 & 6 \\
\hline 15.10 & 410.00 & 48.40 & 566.00 & 385.60 & 2.12 & 4.10 & 16.18 & 7.50 & 7 \\
\hline 15.30 & 345.00 & 68.80 & 491.00 & 440.20 & 1.70 & 22.0 & 16.30 & 7.60 & 8 \\
\hline 16.90 & 396.00 & 54.19 & 495.20 & 450.61 & 3.10 & 16.50 & 15.89 & 7.60 & 9 \\
\hline 18.10 & 354.00 & 111.00 & 363.00 & 526.00 & 27.60 & 8.70 & 18.35 & 7.35 & 10 \\
\hline
\end{tabular}

نفذت تجربة امتز از النحاس (Cu+2) بأخذ $0.5 g$ من كل عينة تزبة ووضعت فـي دورق بلاستيكي حجمه 100ml ، اضيف النحاس بمسستويات

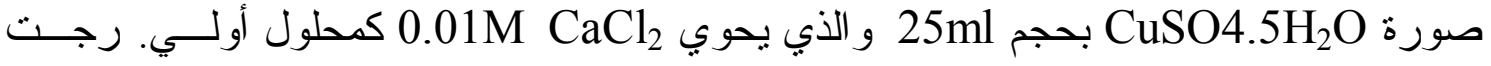

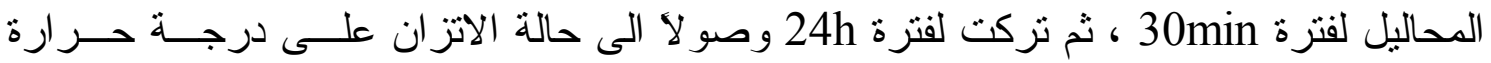

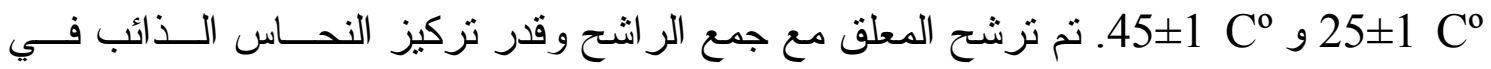

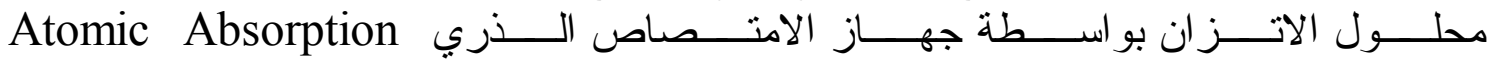
Spectrophotometry (AAS)

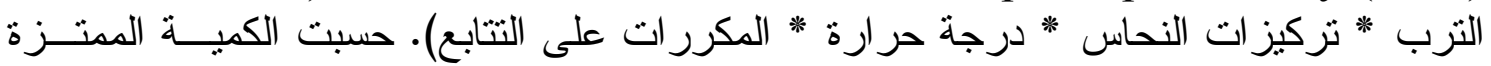
من النحاس من المعادلة التالية: $q=\frac{\left(c_{0}-c\right) v}{W}$ حيث:

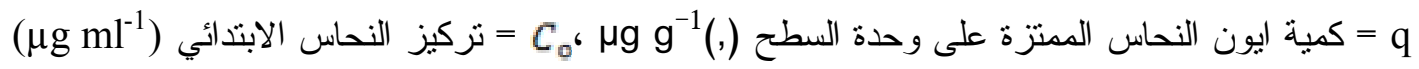
= نركيز النحاس في محلول الاتزان (ml,W) C التربة (g).

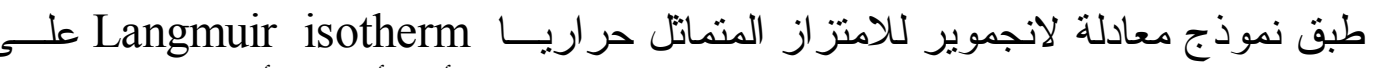

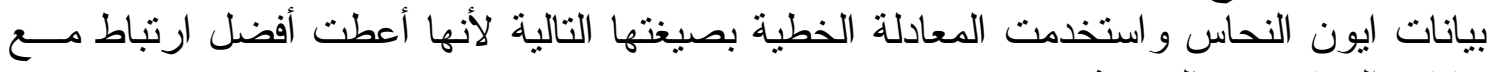
بيانات النحاس في التجربة. $\frac{\mathrm{qe}}{c_{e}}=\mathrm{b} \mathrm{k}_{\mathrm{L}}-\mathrm{k}_{\mathrm{L}} \mathrm{q}_{\mathrm{e}}$

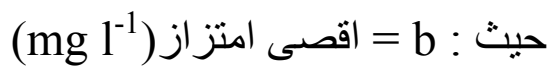
( $\left.\mathrm{g}^{-1}\right)$ = 


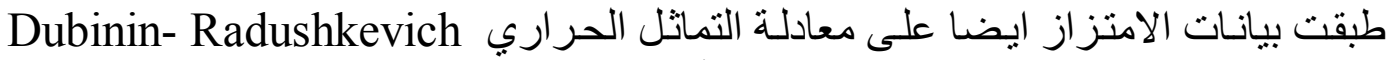

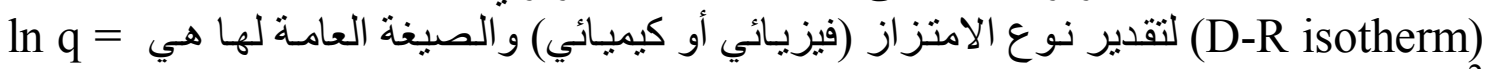
$\operatorname{lnq}_{\mathrm{m}}-\mathrm{k} \square^{2}$

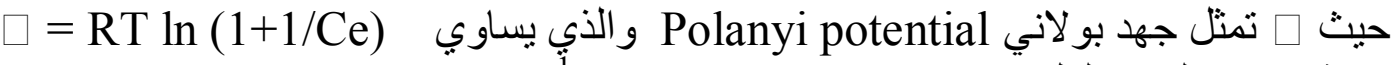

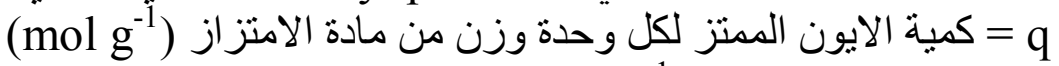
(mol g q $^{-1}$ q

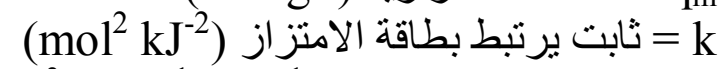

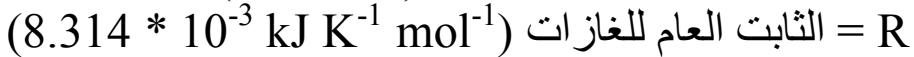

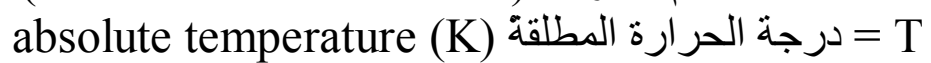

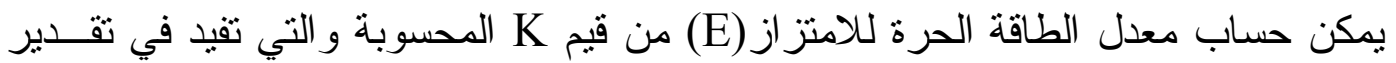
نوع عملية الامتز از من المعادلة التالية:

$$
\mathrm{E}=\frac{1}{\sqrt{-2 K}}
$$

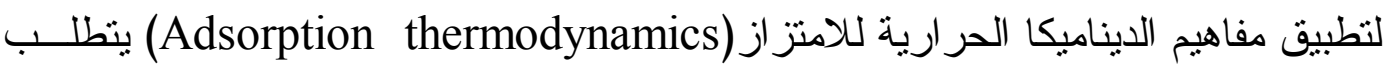

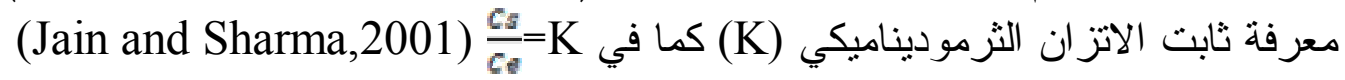
تركيز آيون العنصر الممتز عند الاتزان C C C الاتزان (mg ان

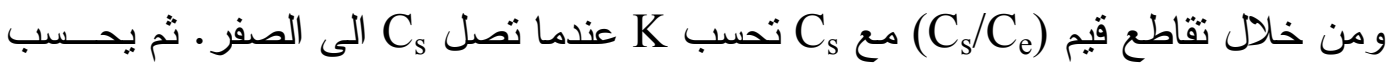

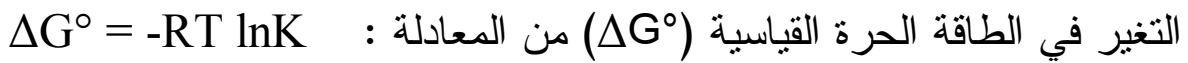
أما التغير في الانثالبي القياسي $\ln \mathrm{K}_{2} / \mathrm{K}_{1}=-\Delta \mathrm{H}^{\circ} / \mathrm{R}\left[1 / \mathrm{T}_{2}-1 / \mathrm{T}_{1}\right]$ $\Delta \mathrm{S}^{\circ}=\left(\Delta \mathrm{H}^{\circ}-\Delta \mathrm{G}^{\circ}\right) / \mathrm{T}$ و يحسب التغير في العشو ائية (الانتروبي النتائج و المناقشة

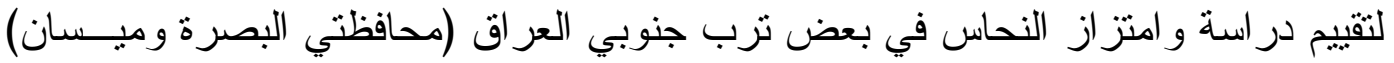

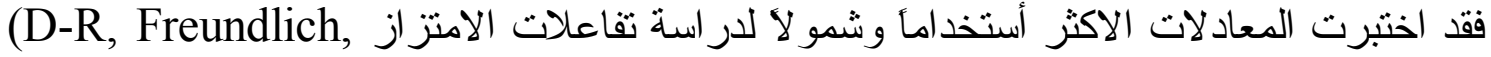
(R2) مرتفعة في وصف طبيعة امتز از (Rangmuir)

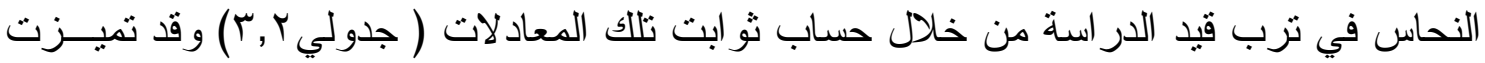

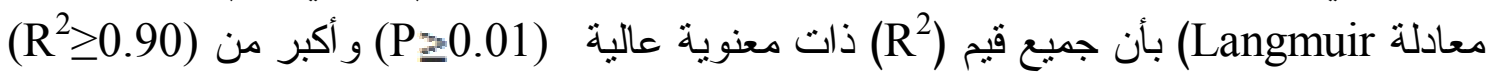

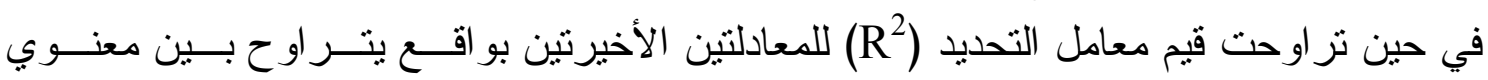

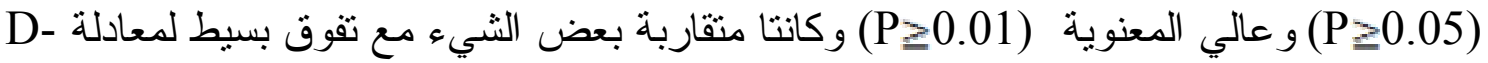

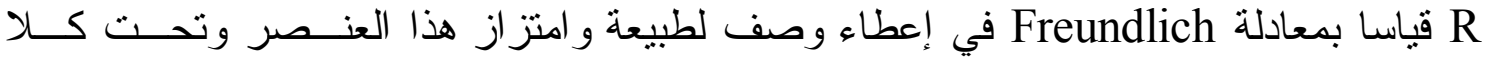

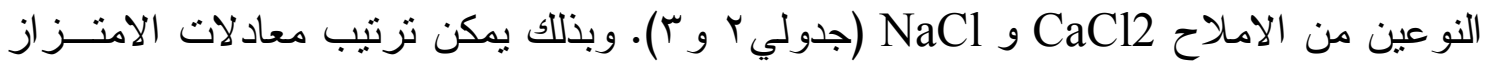

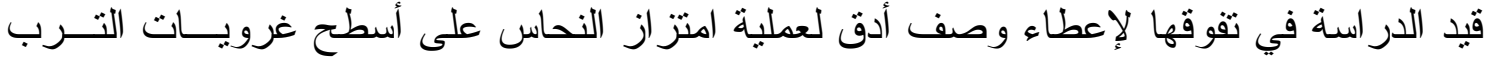

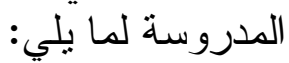




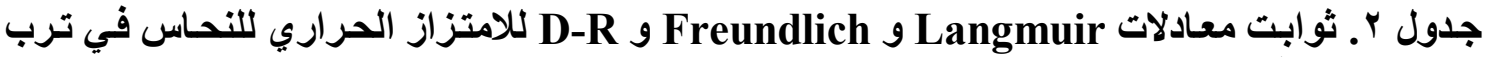
الدارسة في وجود ملح كلوريد الكالسيوم (0.01M CaCl

\begin{tabular}{|c|c|c|c|c|c|c|c|c|c|c|c|}
\hline \multirow[b]{2}{*}{$\mathrm{R}^{2}$} & \multicolumn{2}{|r|}{ D-R } & ثو ابت & \multicolumn{2}{|c|}{ Freundlich } & ثو ابت & \multicolumn{4}{|c|}{ ثوابت Langmuir } & \multirow{2}{*}{ |لتربل } \\
\hline & $\begin{array}{c}\mathrm{E} \\
\mathrm{KJ} \mathrm{mol}^{-1}\end{array}$ & $\begin{array}{l}\mathrm{q}_{\mathrm{m}} \\
\mathrm{ol} \mathrm{g} \mathrm{g}^{-1}\end{array}$ & $\begin{array}{c}\mathrm{k} \\
\mathrm{hol}^{2} \mathrm{KJ}^{-2}\end{array}$ & $\mathrm{R}^{2}$ & $\begin{array}{c}\mathrm{b} \\
\mathrm{Kg} \mathrm{l}^{-1}\end{array}$ & $\begin{array}{c}\mathrm{a} \\
\mathrm{mg} \mathrm{kg}^{-1}\end{array}$ & $\mathrm{R}^{2}$ & $s^{-1}$ & $\begin{array}{c}\mathrm{K}_{\mathrm{L}} \\
1 \mathrm{mg}^{-1}\end{array}$ & $\begin{array}{c}\mathrm{b} \\
\mathrm{mg} \mathrm{kg}^{-1}\end{array}$ & \\
\hline $0.90^{* *}$ & 315 & 00 & 3 & 04 & 399 & .648 & 95 & 10 & 1.43 & 1629.37 & 1 \\
\hline $0.83^{*}$ & 0 & 0 & 9 & 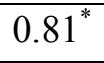 & 3646 & 5 & $90^{* *}$ & 698.63 & 1.30 & 53741 & 2 \\
\hline $0.85^{* *}$ & 13.870 & 3 & 6 & 0.83 & 583 & 42.678 & $0.90^{* *}$ & 1650.01 & .89 & & 3 \\
\hline $0.93^{* *}$ & 0 & 0 & 2 & 2 & 2.876 & 10.165 & $0.90^{* *}$ & & 29 & 604.10 & 4 \\
\hline $0.79^{*}$ & 85 & 050 & 5 & $0.80^{*}$ & 2.333 & 38 & $0.85^{* *}$ & 1 & 1.82 & 00 & 5 \\
\hline $0.88^{* *}$ & 10.870 & 2.032 & & 10 & 3.548 & 111.558 & $0.95^{* *}$ & & 1.25 & 760.62 & 6 \\
\hline $0.80^{*}$ & 15.823 & 553 & & $0.87^{* *}$ & 2.855 & 29 & $0.94^{* *}$ & 00 & 1.41 & 1000.00 & 7 \\
\hline $0.85^{* *}$ & 6 & 3 & & 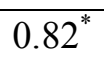 & 2.856 & 724 & 0.9 & & 1.18 & 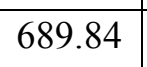 & 8 \\
\hline $0.90^{* *}$ & 772 & 9 & -0.0065 & 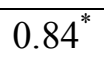 & 4.178 & 93 & $0.95^{* *}$ & 4 & 1.31 & 267 & 9 \\
\hline $0.88^{* *}$ & 628 & 00 & -0.0037 & $0.87^{* *}$ & 3.109 & 772 & $0.95^{* *}$ & 9 & 1.33 & 82.60 & 10 \\
\hline
\end{tabular}

جدول r. ثوابت معادلات Langmuir و و D-R و للامتزاز الحراري للنحاس في ترب الدارسة في وجود ملح كلوريد الصوديوم (0.01M NaCl)

\begin{tabular}{|c|c|c|c|c|c|c|c|c|c|c|c|}
\hline \multirow[b]{2}{*}{$\mathrm{R}^{2}$} & \multicolumn{3}{|c|}{ ثوابت D-R } & \multicolumn{3}{|c|}{ ثوابت Freundlich } & \multicolumn{4}{|c|}{ ثوابت Langmuir } & \multirow{2}{*}{ تنلسل } \\
\hline & $\begin{array}{c}\mathrm{E} \\
\mathrm{KJ} \mathrm{mol}^{-1}\end{array}$ & $\begin{array}{c}\mathrm{q}_{\mathrm{m}} \\
\mathrm{mol} \mathrm{\textrm {g } ^ { - 1 }}\end{array}$ & $\begin{array}{c}\mathrm{k} \\
\mathrm{mol}^{2} \mathrm{KJ}^{-2}\end{array}$ & $\mathrm{R}^{2}$ & $\begin{array}{c}\mathrm{b} \\
\mathrm{Kg} \mathrm{l}^{-1}\end{array}$ & $\begin{array}{c}\mathrm{a} \\
\mathrm{mg} \mathrm{kg}^{-1}\end{array}$ & $\mathrm{R}^{2}$ & $\begin{array}{l}\text { MBC } \\
1 \mathrm{Kg}^{-1}\end{array}$ & $\begin{array}{c}\mathrm{K}_{\mathrm{L}} \\
1 \mathrm{mg}^{-1}\end{array}$ & $\begin{array}{c}\mathrm{b} \\
\mathrm{mg} \mathrm{kg}^{-1}\end{array}$ & \\
\hline $0.97^{* *}$ & 8.703 & 3.163 & -0.0066 & $0.99^{* *}$ & 4.088 & 419.856 & $0.96^{* *}$ & 6220.28 & 2.68 & 2321.68 & 1 \\
\hline $0.99^{* *}$ & 11.627 & 1.530 & -0.0037 & $0.82^{*}$ & 2.744 & 11.663 & $0.85^{* *}$ & 667.01 & \begin{tabular}{|l|}
1.06 \\
\end{tabular} & 629.25 & 2 \\
\hline $0.84^{*}$ & 8.278 & 4.150 & -0.0073 & $0.88^{* *}$ & 4.315 & 1206.980 & $0.90^{* *}$ & 1805.56 & 1.75 & \begin{tabular}{|l|}
1031.75 \\
\end{tabular} & 3 \\
\hline $0.81^{*}$ & 11.186 & 1.230 & -0.0040 & $0.83^{*}$ & 2.819 & 11.202 & $0.95^{* *}$ & 116.90 & 1.75 & \begin{tabular}{|l|}
638.23 \\
\end{tabular} & 4 \\
\hline $0.91^{* *}$ & 10.917 & 2.700 & -0.0042 & $0.90^{* *}$ & 2.771 & 84.120 & $0.98^{* *}$ & 2476.70 & 2.36 & $\mid 1049.45$ & 5 \\
\hline $0.93^{* *}$ & 10.204 & 2.300 & -0.0048 & $0.83^{*}$ & 3.185 & 103.944 & $0.85^{* *}$ & 929.69 & 1.21 & 768.34 & 6 \\
\hline $0.88^{* *}$ & 12.903 & 1.880 & -0.0030 & $0.86^{* *}$ & 3.114 & 13.684 & $0.90^{* *}$ & 2475.62 & 2.17 & 1140.84 & 7 \\
\hline $0.92^{* *}$ & 10.000 & 2.550 & -0.0050 & $0.76^{*}$ & 3.624 & 195.344 & $0.90^{* *}$ & 1155.35 & 1.45 & 796.79 & 8 \\
\hline $0.94^{* *}$ & 8.006 & 4.980 & -0.0078 & $0.84^{*}$ & 4.036 & 1308.278 & $0.95^{* *}$ & 369.08 & 1.17 & 315.45 & 9 \\
\hline $0.84^{*}$ & 8.842 & 3.685 & -0.0064 & $0.82^{*}$ & 3.577 & 765.420 & $0.90^{* *}$ & 273.45 & 1.03 & 265.49 & 10 \\
\hline
\end{tabular}

Langmuir equation > D-R equation >Freundlich للسعة الامتزازية (أقصى امتزاز b) الذي يمثل احد ثوابت معادلة Langmuir من - 182.60)

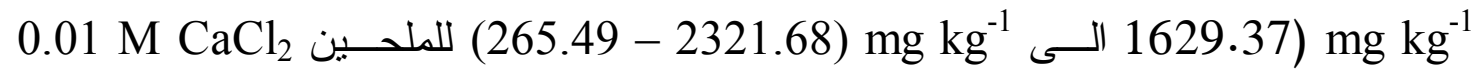

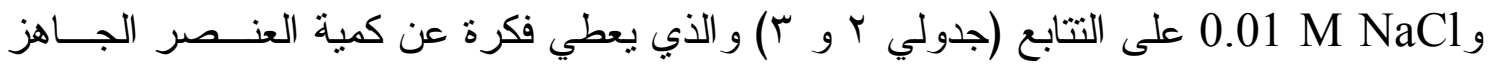

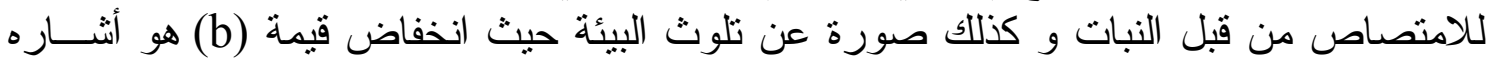

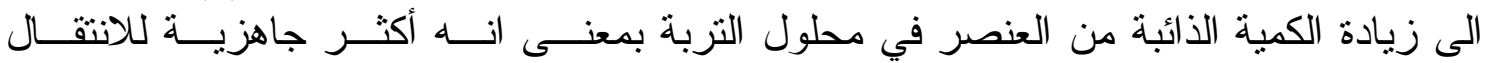

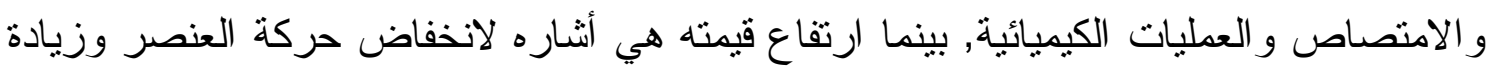




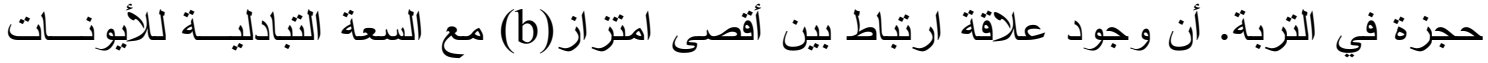

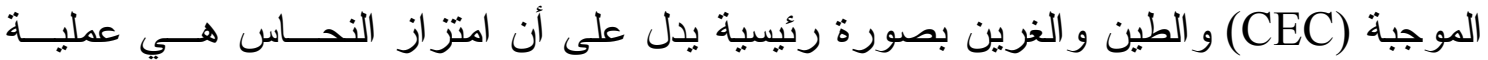

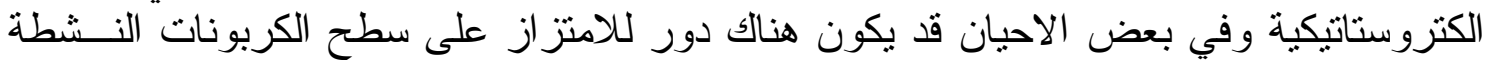

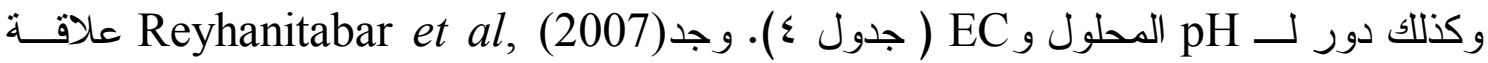

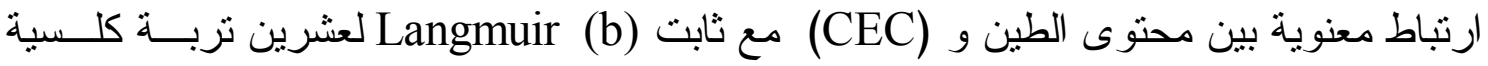

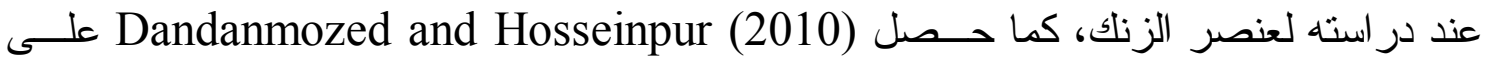

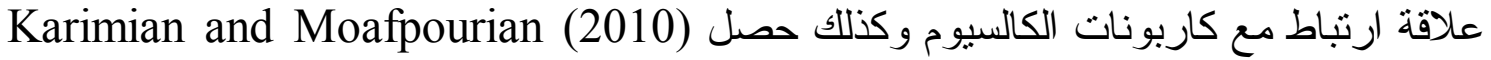

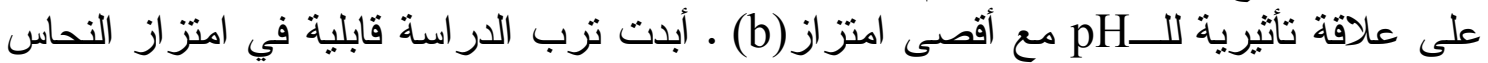

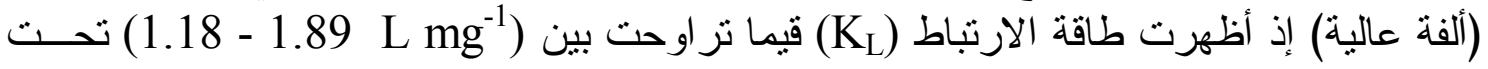
ظروف

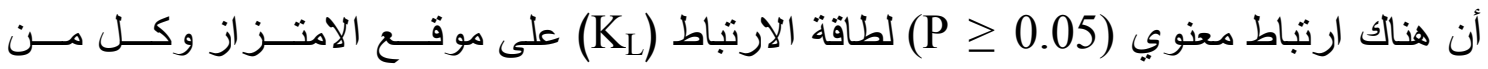

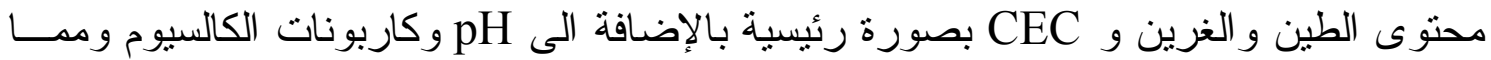

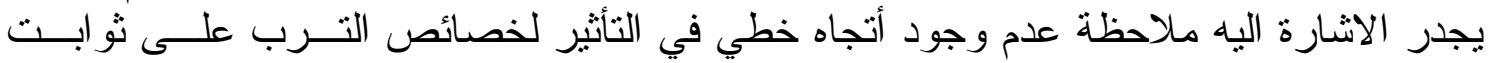

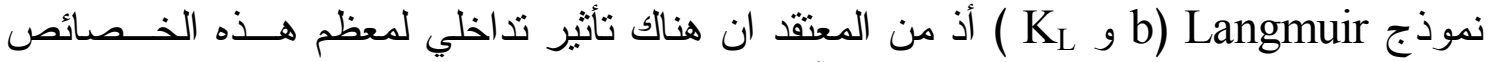

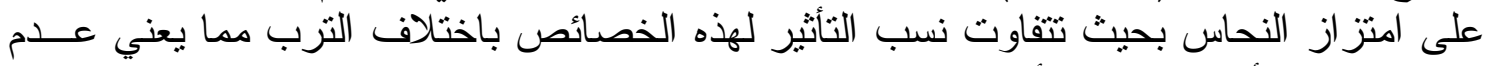

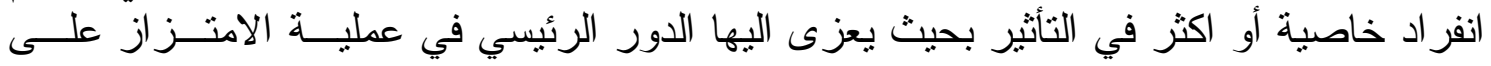

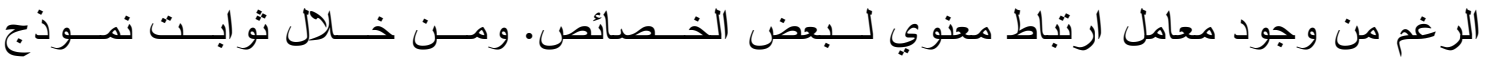

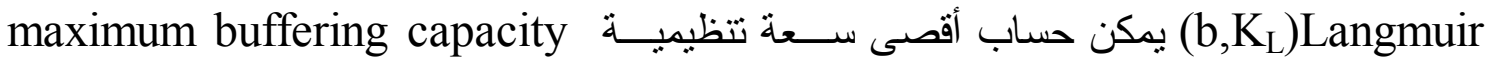
وقد تر اوحت قيمتها في الترب المدروســة بـين (MBC) وجود

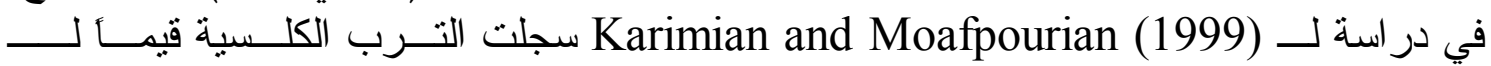

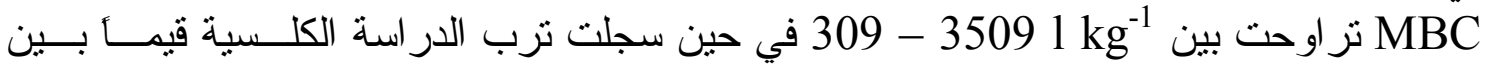

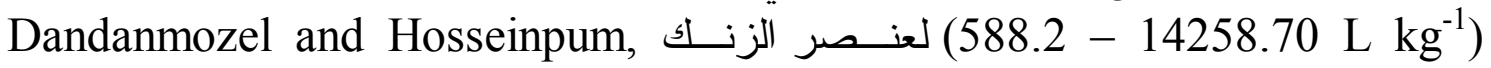

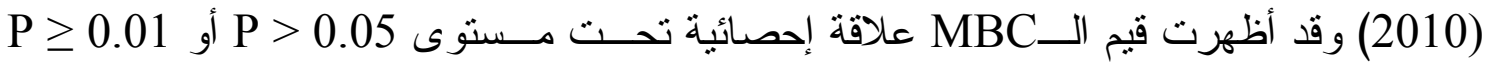

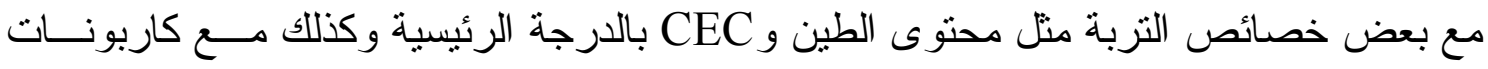

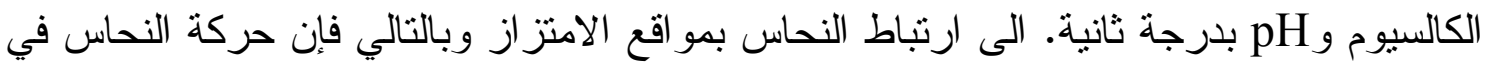

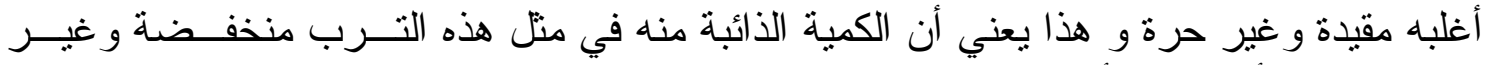

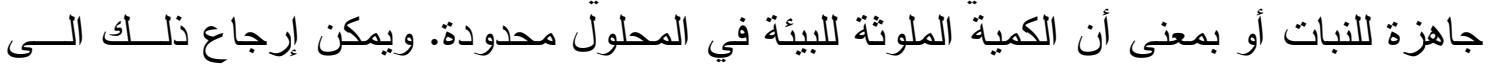

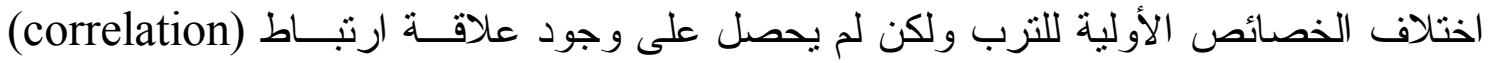

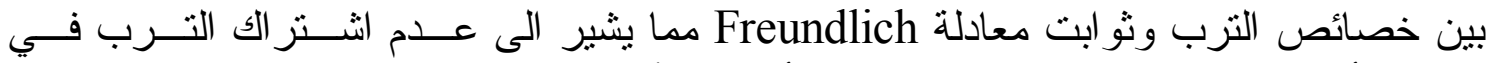

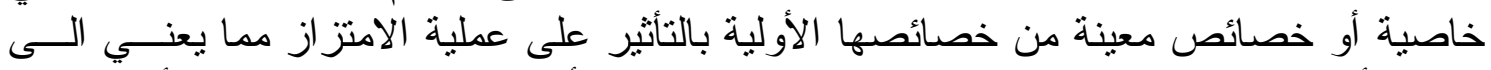

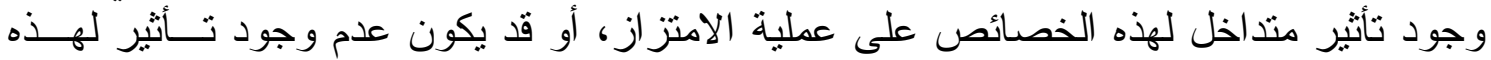

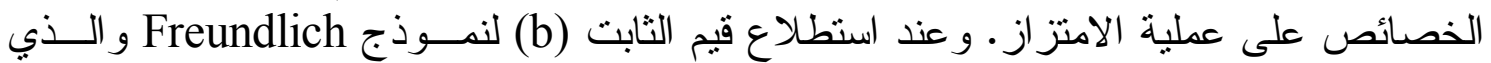

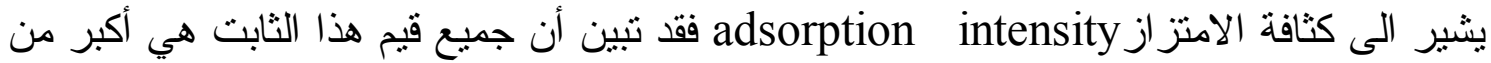

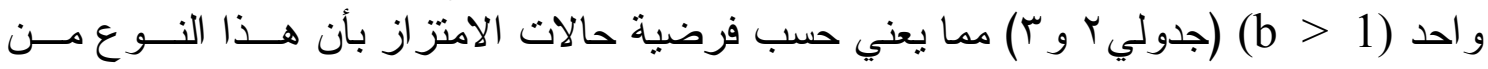

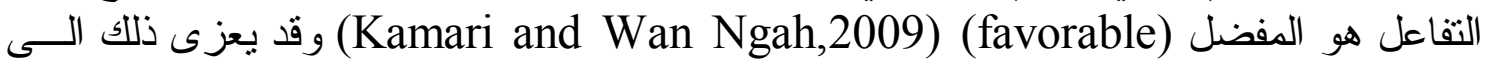

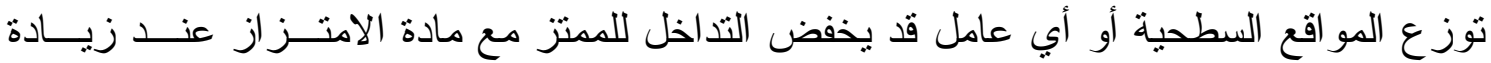

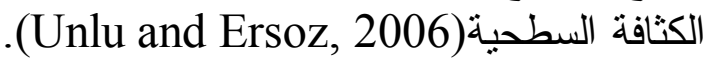




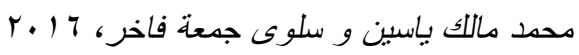

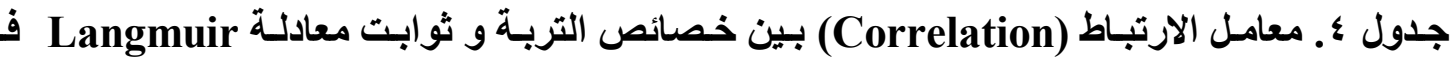

وجود الملحين كلوريد الكالسيوم وكلوريد الصوديوم (0.01 M NaCl 0.01 M CaCl

\begin{tabular}{|c|c|c|c|c|c|c|}
\hline \multicolumn{3}{|c|}{$\begin{array}{c}0.01 \mathrm{M} \\
\mathrm{NaCl}\end{array}$} & \multicolumn{3}{|c|}{$\begin{array}{c}0.01 \mathrm{M} \\
\mathrm{CaCl}_{2}\end{array}$} & \multirow[t]{2}{*}{ خصائص } \\
\hline $\mathrm{MBC}$ & $\mathrm{K}_{\mathrm{L}}$ & b & $\mathrm{MBC}$ & $\mathrm{K}_{\mathrm{L}}$ & B & \\
\hline $0.765^{*}$ & $0.672^{*}$ & $0.582^{*}$ & -0.297 & -0.190 & -0.261 & $\mathrm{CaCO}_{3}$ \\
\hline $0.926^{* *}$ & $0.909^{*}$ & $0.680^{*}$ & $0.716^{*}$ & $0.596^{*}$ & $0.511^{*}$ & Clay \\
\hline 0.362 & $0.500^{*}$ & $0.680^{*}$ & 0.480 & $0.565^{*}$ & $0.524^{*}$ & Silt \\
\hline-0.235 & -0.391 & -0.354 & -0.323 & 0.019 & -0.389 & O.M \\
\hline $0.832^{* *}$ & $0.672^{*}$ & $0.740^{*}$ & $0.598^{*}$ & $0.710^{*}$ & $0.619^{*}$ & CEC \\
\hline 0.422 & 0.397 & -0.386 & $0.616^{*}$ & $0.505^{*}$ & $0.505^{*}$ & $\mathrm{pH}$ \\
\hline $0.507^{*}$ & $0.506^{*}$ & $0.549^{*}$ & -0.297 & -0.124 & -0.312 & EC \\
\hline
\end{tabular}

بعد تطبيق نموذج Freundlich للامنز از الحراري على نتائج امنزاز النحاس في تــرب

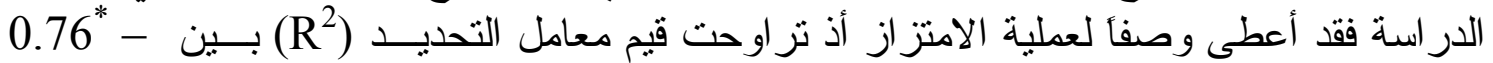

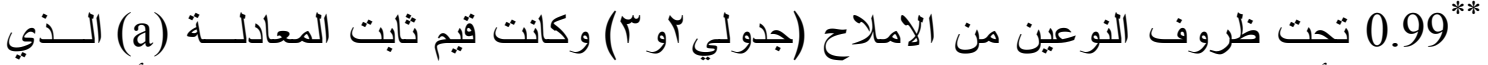

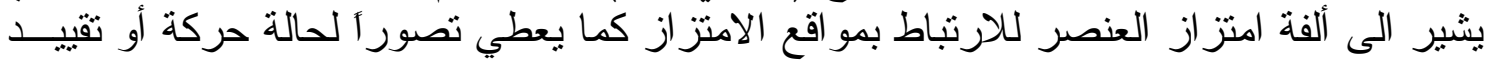

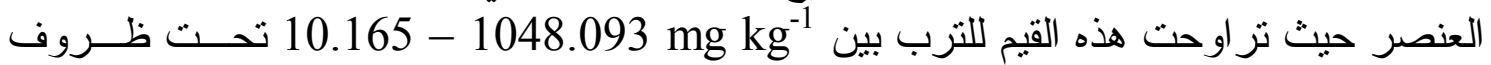

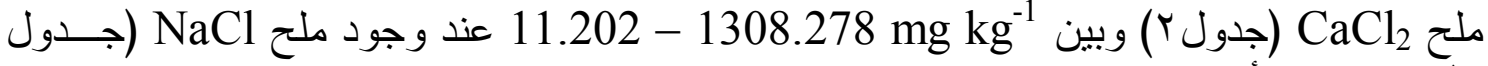

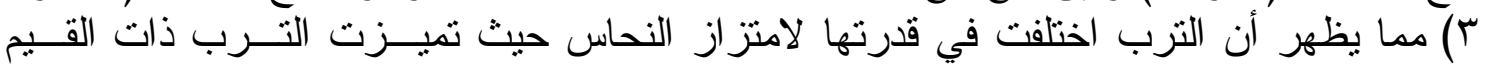

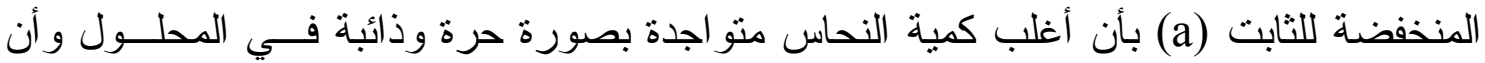

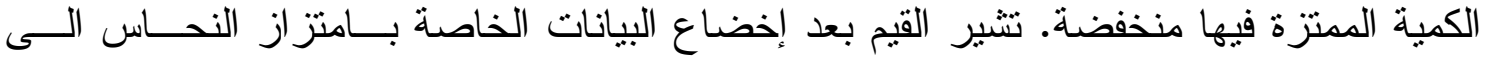

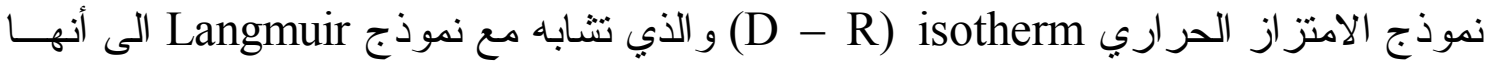

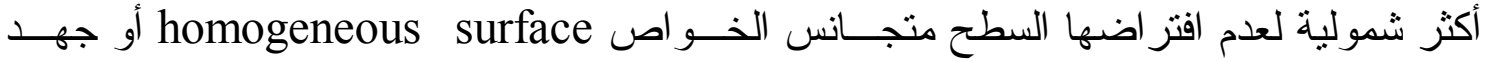

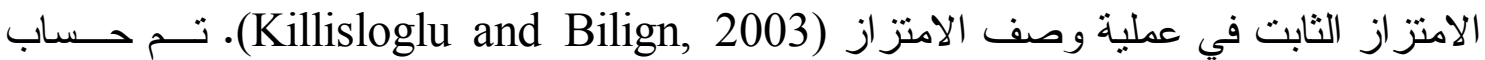

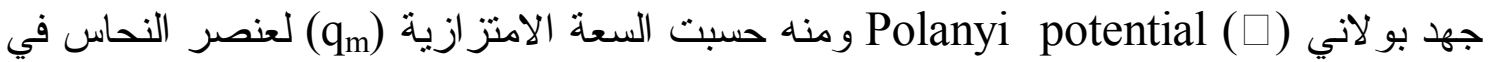
الترب قيد الدر استة والتي تز اوحت بين

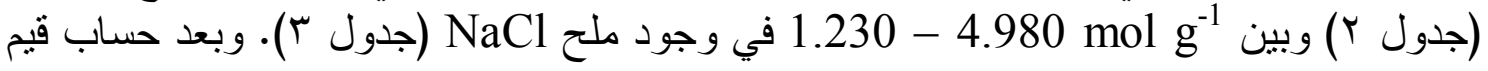
ثابت k لنموذج (R - R) و التي ترتبط مع طاقة الامتز از فقد تز اوحت بقيم 0.0065 - 0.0020-)

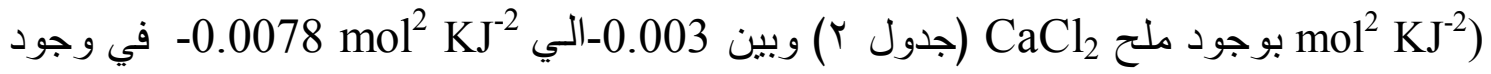

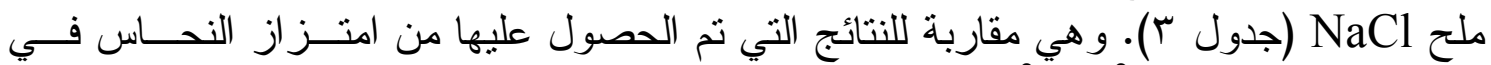

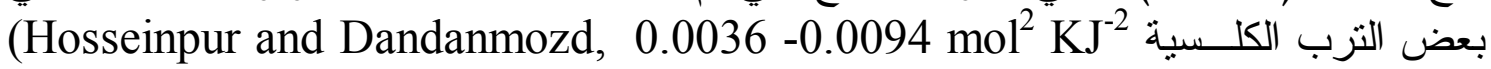
.2010)

تعبر قيم (E) عن معدل الطاقة الحرة للتفاعل وتقيد في تقدير نوع عملية الامتـز از فــاذا كانت قيمها

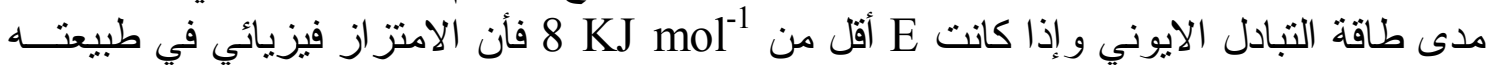

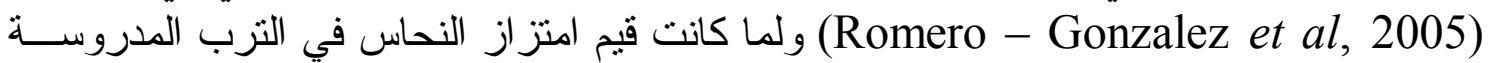

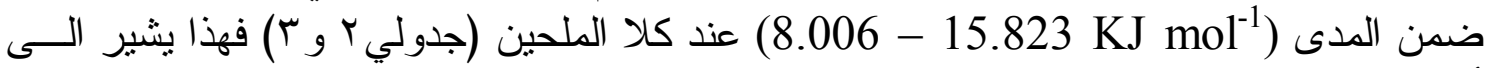

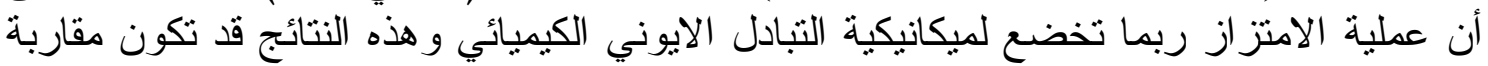

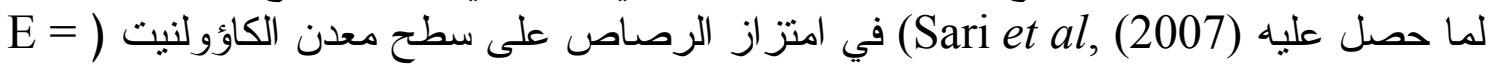




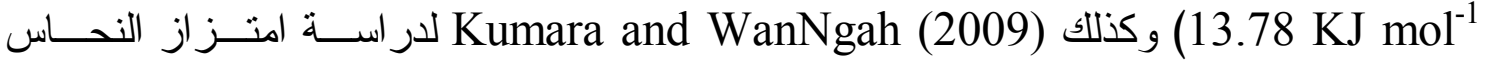

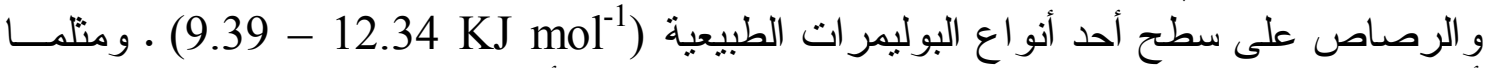

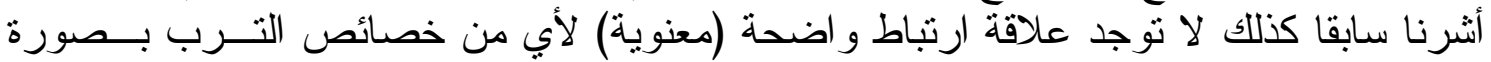

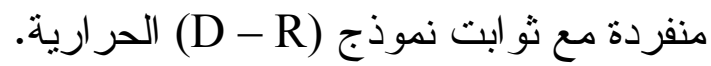
( في العديد من التطبيةــات أمــر أ

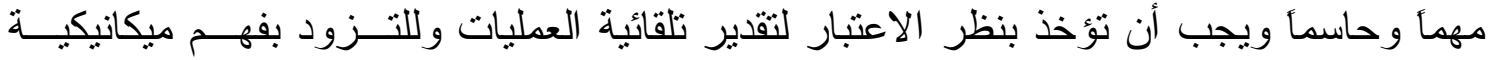

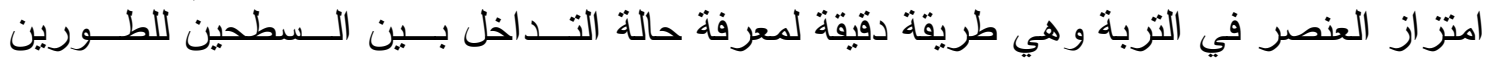
الصلب و السائل (Zubieta et al, 2008) فقد أثشارت البيانات (جدولي ه و 7) أن معامل الاتز ان

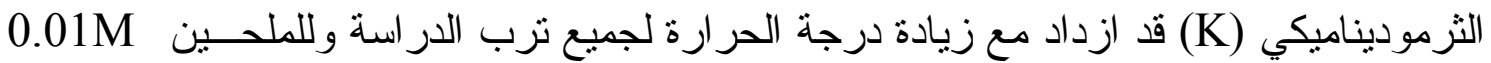

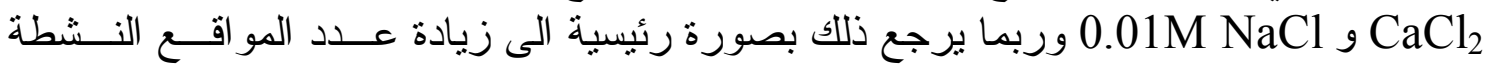

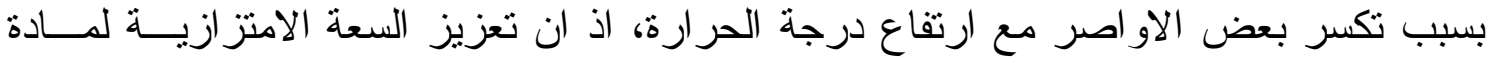

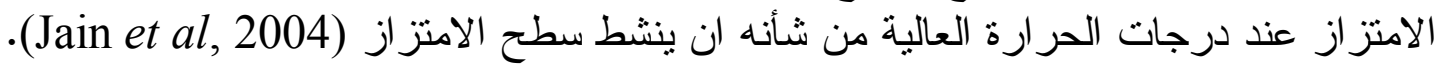

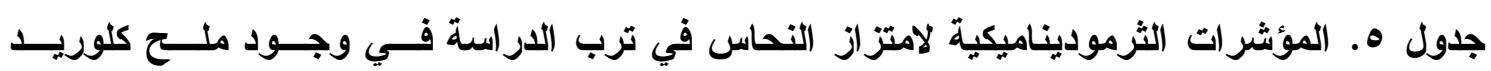

الكالسيوم العثرت ت

\begin{tabular}{|c|c|c|c|c|c|c|c|}
\hline \multicolumn{2}{|c|}{$\Delta \mathrm{S}^{\circ} \mathrm{J} \mathrm{mol}^{-1}$} & \multirow[t]{2}{*}{$\Delta \mathrm{H}^{\circ} \mathrm{KJ} \mathrm{mol}^{-1}$} & \multicolumn{2}{|c|}{$\Delta G^{\circ} \mathrm{kJ} \mathrm{mol}^{-1}$} & \multicolumn{2}{|c|}{ K } & \multirow{2}{*}{ الترب } \\
\hline 45 & 25 & & 45 & 25 & 45 & 25 & \\
\hline 55.378 & 55.403 & 1.199 & -16.42 & -15.32 & 496.12 & 481.47 & 1 \\
\hline 58.065 & 58.069 & 1.944 & -16.53 & -15.37 & 517.70 & 493.14 & 2 \\
\hline 64.750 & 64.767 & 4.121 & -16.48 & -15.19 & 507.46 & 457.78 & 3 \\
\hline 65.524 & 65.525 & 4.407 & -16.44 & -15.13 & 499.80 & 447.66 & 4 \\
\hline 70.062 & 70.066 & 5.861 & -16.43 & -15.03 & 497.86 & 430.00 & 5 \\
\hline 52.612 & 52.619 & 0.299 & -16.44 & -15.39 & 500.09 & 496.37 & 6 \\
\hline 52.166 & 52.177 & 0.147 & -16.45 & -15.41 & 501.23 & 499.39 & 7 \\
\hline 52.615 & 52.757 & 0.320 & -16.42 & -15.41 & 500.13 & 496.15 & 8 \\
\hline 51.927 & 51.922 & 0.081 & -16.44 & -15.40 & 499.47 & 498.46 & 9 \\
\hline 60.136 & 60.749 & 2.873 & -16.26 & -15.24 & 501.02 & 466.30 & 10 \\
\hline
\end{tabular}

جدول T. المؤشرات الثرموديناميكية لامتزاز النحاس في ترب الدراسة في وجود ملح كلوريد الصوديوم

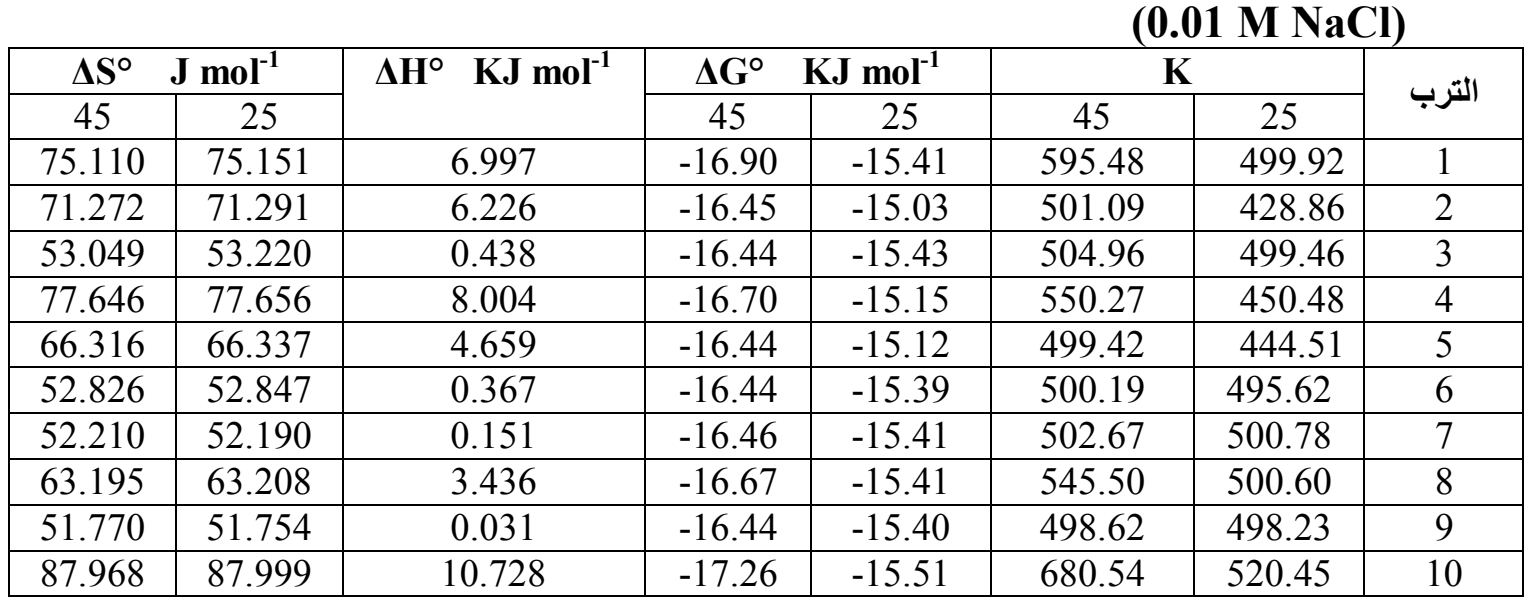




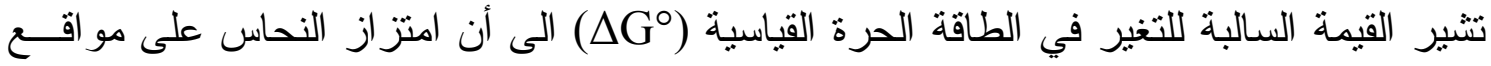

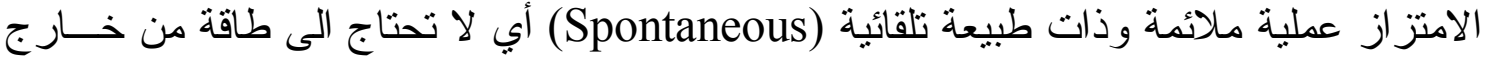

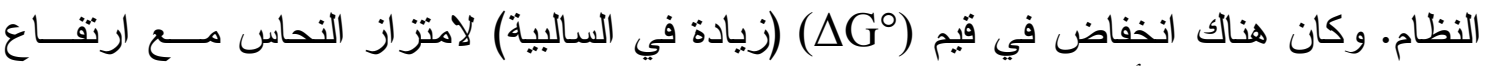

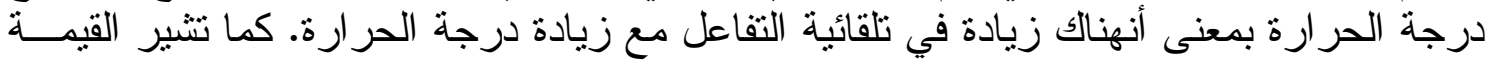

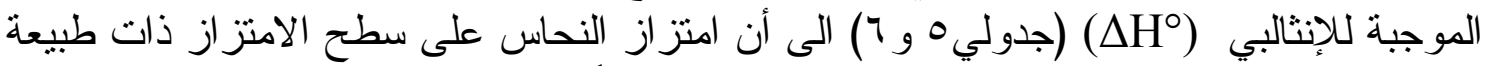

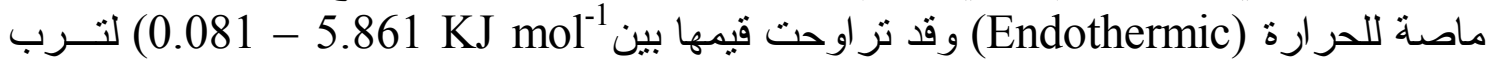

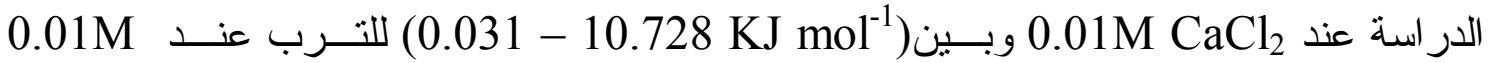
NaCl

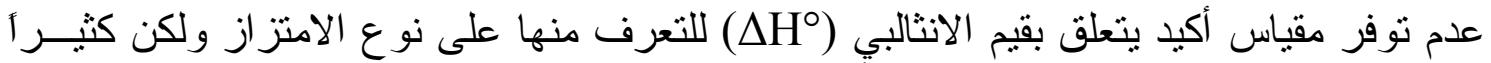

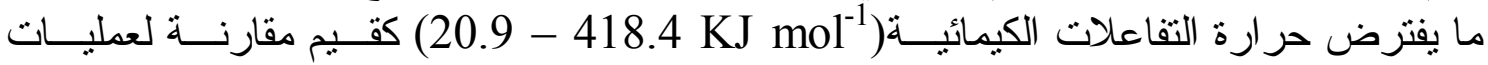

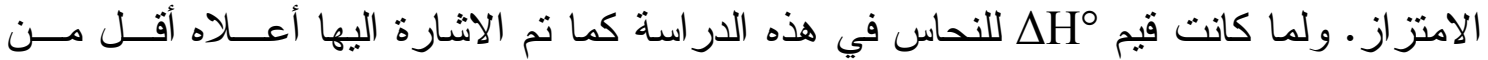

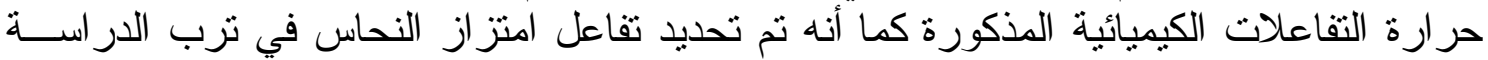

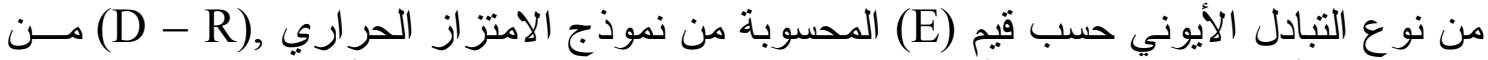

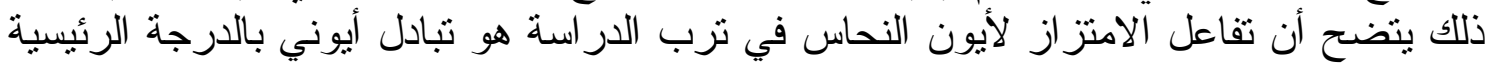

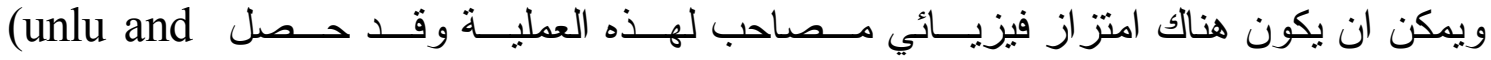
Erosoz,2006)

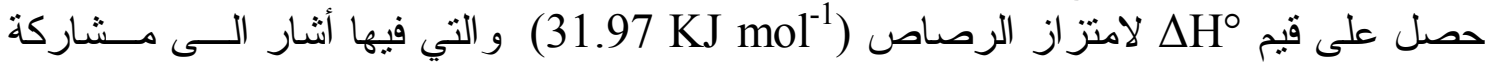
الامتز از الكيميائي chemisorption (وهو نوع من الامتز از يتضمن تفاعل كيميائي بين السطح

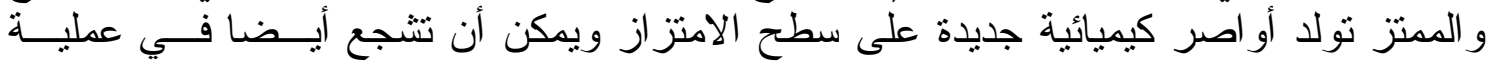

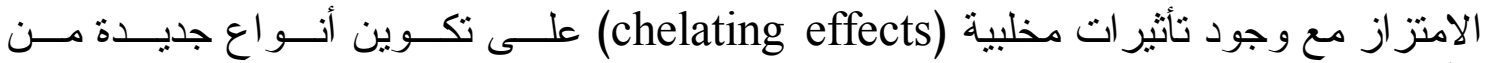

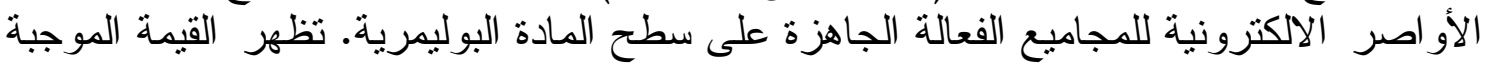

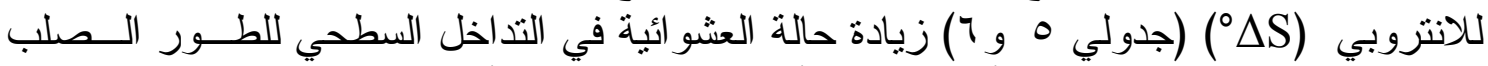

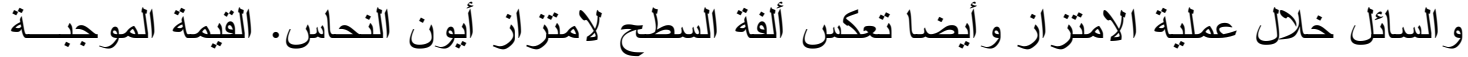
للانتروبي (

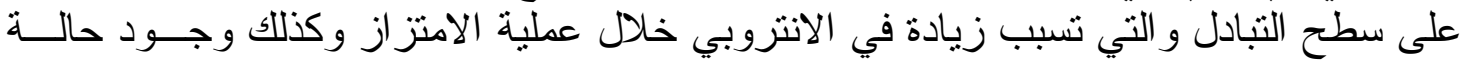

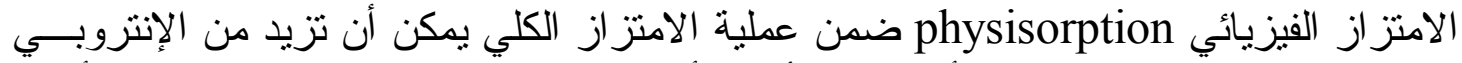

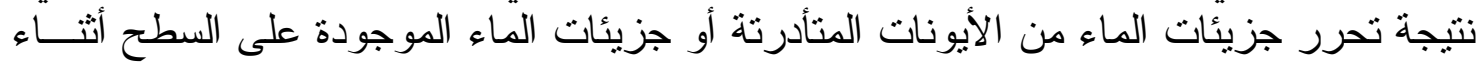

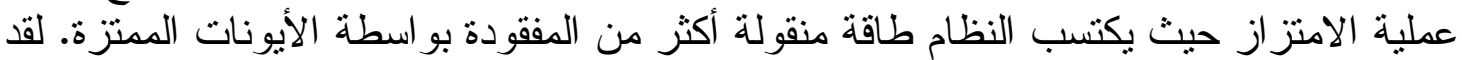

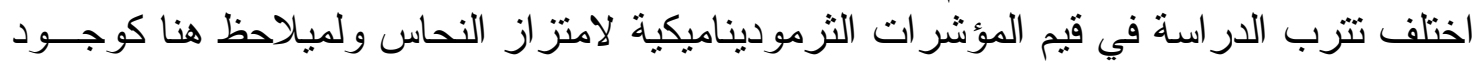
علاقة ارتباط و اضحة بين خصائص نرب الدر اسة و المؤشر ات الثرموديناميكية عند كلا الملحين ماعدا محتوى الترب من المادة العضوية مع جميع المفردات الثرموديناميكية عند وجود NaCl

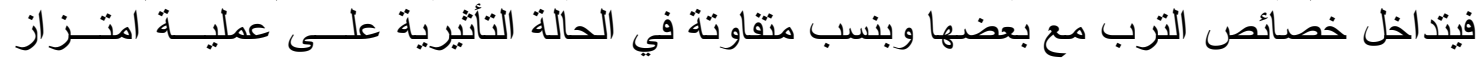

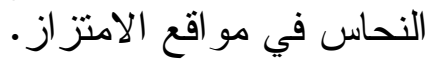

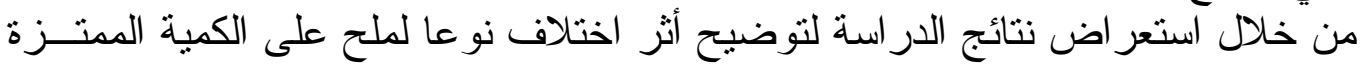

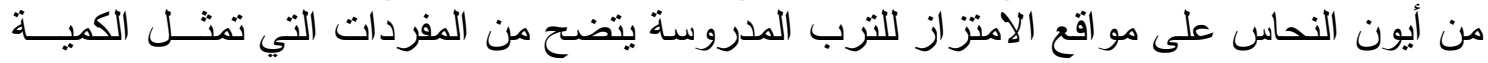

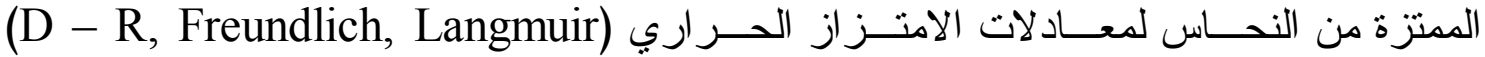

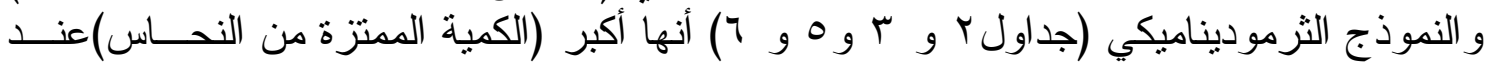
وجود ملح كلوريد الصوديوم (NaCl) في محلول التربة الاولي قياسأ بالملح كلوريد الكالـسيوم 
$\left.\mathrm{Cu}^{+2} \mathrm{Ca}^{+2} \mathrm{Ca}^{+} \mathrm{Na}^{+} \mathrm{CaCl}_{2}\right)$

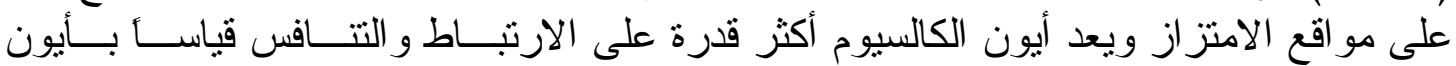

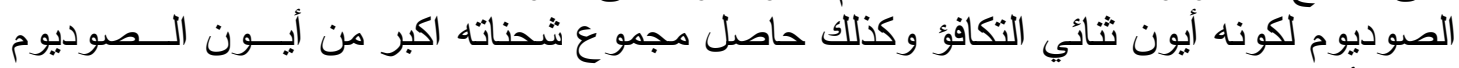

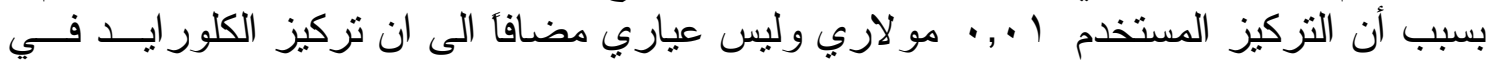
ملح

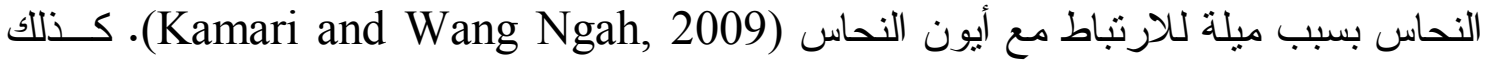

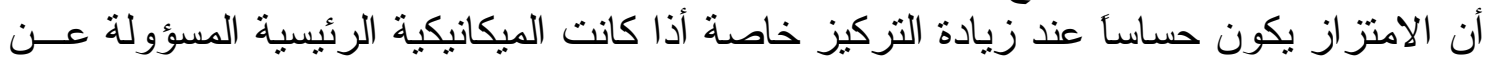

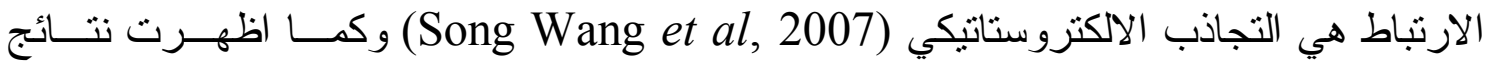

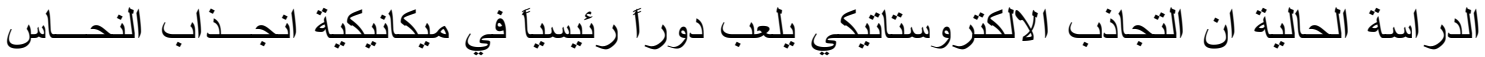

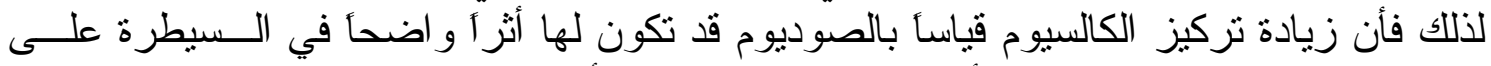

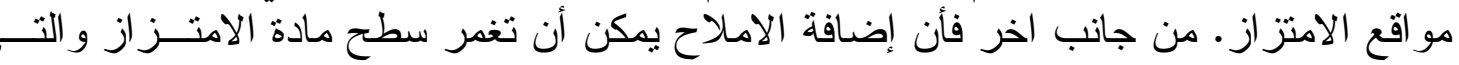

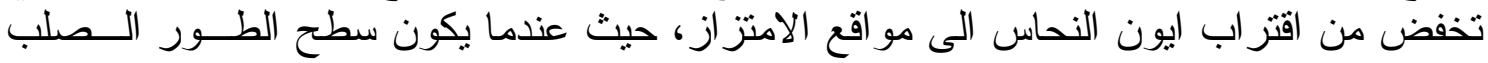

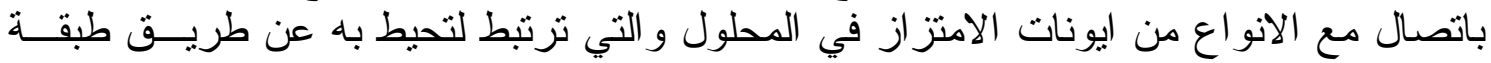

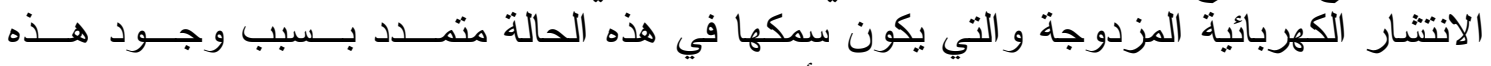

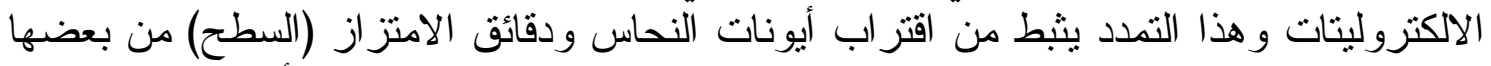

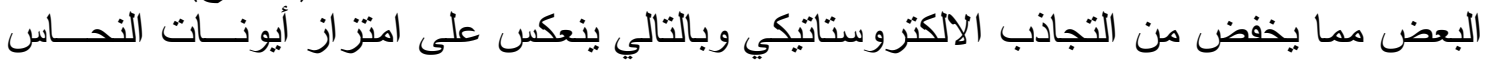
.(Krishnan and Anirudhan, 2003)

جدول V. معامل الارتباط (Correlation) بين خصائص التربة والمؤشرات الثرموديناميكية في وجود

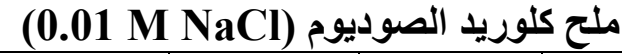

\begin{tabular}{|c|c|c|c|c|c|c|c|}
\hline $\begin{array}{c}\Delta \mathrm{S}^{\circ} \\
45\end{array}$ & $\begin{array}{c}\Delta S^{\circ} \\
25\end{array}$ & $\Delta \mathbf{H}^{\circ}$ & $\begin{array}{c}\Delta \mathbf{G}^{\circ} \\
\mathbf{4 5}\end{array}$ & $\begin{array}{c}\Delta \mathbf{G}^{\circ} \\
25\end{array}$ & $\begin{array}{l}\mathrm{K} \\
45\end{array}$ & $\begin{array}{l}K \\
25\end{array}$ & خصائص \\
\hline 0.190 & 0.188 & 0.193 & -0.103 & 0.169 & 0.082 & -0.149 & $\mathrm{CaCO}_{3}$ \\
\hline 0.489 & 0.489 & 0.490 & -0.377 & 0.118 & 0.376 & -0.113 & Clay \\
\hline 0.106 & 0.105 & 0.112 & -0.003 & 0.167 & -0.013 & -0.164 & $\begin{array}{l}\text { Silt } \\
\end{array}$ \\
\hline $0.793^{*}$ & $0.794^{*}$ & $0.792^{*}$ & $-0.650^{*}$ & 0.130 & $0.668^{*}$ & -0.116 & O.M \\
\hline 0.440 & 0.440 & 0.431 & -0.484 & -0.132 & 0.465 & 0.139 & CEC \\
\hline 0.004 & 0.005 & 0.033 & $0.641^{*}$ & $0.641^{*}$ & -0.392 & -0.661 & $\mathrm{pH}$ \\
\hline-0.031 & -0.031 & -0.039 & -0.085 & -0.185 & 0.070 & 0.190 & $\mathrm{EC}$ \\
\hline
\end{tabular}

Baker, D. E. (1990). Heavy metals in soils. Copper.,B.J. Alloway (Ed.). Blackie and Sons Ltd., London.151-176.

Black, C.A. (1965). Methods of soil analysis. Part1. Physical properties. Wisconsin, Madison, American society, Agronomy, Inc. Publisher.

Chen, J.C.; M. Y. Wey and Z. S. Liu (2001). Adsorption mechanism of heavy metalson sorbent during incineration. Journal of Environmental Engineering, 127: 63-69. 
Dali - Yousef, N.; B. Oddane and Z. Drriche (2006). Adsorption of zinc on natural sediment of Tacna River. Journal of Hazardous Materials, 137:1263-1270.

Dandanmozd, F. and A.R. Hossinpur (2010). Thermodynamic parameters of zinc sorption in some calcareous soils. Journal of American Science, 6 (7):298304.

Hossinpur, A.R. and F. Dandanmozd (2010). Sorption characteristics of Copper in some calcareous soils of western Iran. Journal of American Science, 6 (11):103-108.

Jain, C.K. and M.K. Sharma (2002). Adsorption of Cadmium on bed sediment of River Hindon: adsorption models and kinetics. Water, Air and Soil Pollution, 137:1-19.

Jain, C.K.; D.C. Singhal and M.K. Sharma (2004). Adsorption of zinc on bed sediment of River Hindan: adsorption models and kinetics. Journal of Hazardous Materials, 114:231-239.

Kamari, A. and W.S. Wan Ngah (2009). Isotherm, kinetic and thermodynamic studies of lead and copper uptake by $\mathrm{H}_{2} \mathrm{SO}_{4}$ modified chitosan. Collides and Surfaces B: Biointerfaces, 73:257-266.

Karimian, N. and G.R. Moafpouryan (1999). Zinc adsorption characteristics of selected soil of Iranand their relationship with soil properties. Communication in Soil Science and plant Analysis, 30:1721-1731.

Kilislioglu, A. and B. Bilgin (2003). Thermodynamic and kinetic investigations of uranium adsorption amber lite IR-118H resin. Applied Radiation and Isotopes, 58:155-160.

Krishnan, K.A and T.S. Anirudhan (2003). Removal of cadmium(II) from aqueous solution by steam- activated sulphurised carbon prepared from sugarcane bagasse pith: Kinetics and equilibrium studies. Water SA 29:147156.

Li, Q.; J. Zhai; W.zhang; M.Wang and J.zhou (2007). Kinetics studies of adsorption of $\mathrm{pb}(\mathrm{II}), \mathrm{Cr}(\mathrm{III})$ and $\mathrm{Cu}(\mathrm{II})$ from aqueous solution by sawdust and modified peanut husk, Journal of Hazardous Materials, 141:163-167.

Lopez; E., B. soto; M. Arias; D.Rubinos and M.T. Barral (1998). Adsorption properties of red mud and its use for waste water treatment. Water Research, 10:1-155.

Mansour, M.S.; M.E. Ossman and H.A. Farag (2011). Removal of Cd (II) ion from waste water by adsorption onto polyaniline coated on sawdust. Desalination, 272: 301-305.

Mills, A.C. and G.W.Biggar (1969a). Adsorption of gamma BHC from solution on sevsral selected adsorbent. Journal of Agriculture and Food Chemistry, 17: 604-608.

Mills, A.C. and G.W.Biggar (1969b). Adsorption of Hexachloro cyclohexane from solutions. The differential heat of adsorption applied to adsorption from dilute solutions on organic and inorganic surfaces. Journal of Collide Science, 29: 720-731. 
Mills, A.C. and G.W.Biggar (1969c). Solubility temperature effect on the adsorption of gamma andbeta BHC from aqueous and hexane solutions by soil materials. Soil Science Society of America Proceeding, 33: 210-216.

Page, A.L.,R.H. Miller and D.R.Keeney (1982). Methods of soil analysis, part(2) $2^{\text {nd }}$ ed. Agronomy 9-Wisconsin, Madison. American Society Agronomy. Inc. Publisher.

Reyhanitabar, A.; N.Karimian; M.Ardalan, G. Savaghebi and M. Ghannadha (2007). Comparison of five adsorption isotherms for predication of zinc retention in calcareous soils and the relationship of their coefficient with soil characteristics. Communication in Soil Science and Plant Analysis, 38: 147-158.

Romero-Gonzalez, J.; J.R. Peralta-videa; E.Rodriguez; S.L. Ramirez and J.L, Gardea-Torresdey (2005). Determination of thermodynamic parameters of $\mathrm{Cr}$ (VI) adsorption from aqueous solution onto Agave lechuguilla biomass. Journal Chemistry Thermodynamics, 37:343-347.

Sari, A.; M. Tuzen; D. Citak and M. soylak (2007). Equilibrium, kinetic and thermodynamic studies of adsorption of $\mathrm{Pb}$ (II) from aqueous solution onto Turkish kaolinite clay. Journal of Hazardous Materials, 149: 283-291.

Sawyer, C.N. and P.L. Mccarty (1978). Chemistry for environmental engineering. $3^{\text {rd }}$ ed., McGraw-Hill, Singapore, 58-90.

Song Wang, X.; J. Huang; H. Qing Hu; J. Wang and Y. Qin (2007). Determination of kinetic and equilibrium parameter of the batch adsorption of Ni (II) from aqueous solutions by Na-mordenite. Journal of Hazardous Materials, 142: 468-476.

Sposito,G.(1984). The surface chemistry of soils. Oxford University Press, Newyork.

Srivastava, V. C.; I.D. Mall and L.M. Mishra (2006). Characterization of mesoporous rice husk ash (RHA) and adsorption kinetics of metal ions from aqueous solution onto RHA. Journal of Hazardous Materials, 134: 257-267.

Unlu, N. and M. Ersoz (2006). Adsorption characteristics of heavy metal ions onto a low cost biopolymeric sorbent from aqueous solutions. Journal of Hazardous Materials, 136: 272-280.

Zubieta, C.E.; P. V. Messina; C.L. Luengo; M.Dennehy; O.Pieroni and P.C.Schulz (2008). Reactive dyes remotion by porous $\mathrm{TiO}_{2}$-chitosan materials. Journal of Hazardous Materials, 152: 765-777. 
محد مالك باسبن و سلوى جمعة فاخر ، 7 ب م

\title{
Using the Equilibrium and Thermodynamic Parameters of Copper Adsorption in some Calcareous Soils
}

\section{Mohammed M. Yassen and Salwa J. Fakher}

Department of Soil Sciences and Water Resources / Agriculture College / University of

Basrah - Iraq

\begin{abstract}
Ten different soil samples from Basrah and Messan governorates of southern Iraqi were used to study the mechanism of copper $(\mathrm{Cu})$ adsorption. Copper was added at $0,4,8,16$ and $32 \mathrm{mg} \mathrm{Cu} \mathrm{kg}^{-1}$ soil and two salts $\left(0.01 \mathrm{M} \mathrm{CaCl}_{2}\right.$ and $\left.0.01 \mathrm{M} \mathrm{NaCl}\right)$ with two temperature $\left(25 \pm 1 \mathrm{C}^{\circ}\right.$ and $\left.45 \pm 1 \mathrm{C}^{\circ}\right)$. Copper adsorption was tested using isothermal equation parameters of Langmuir, Freundlich and D-R as well as thermodynamic parameters $\left(\mathrm{K}^{\circ}, \Delta \mathrm{G}^{\circ}, \Delta \mathrm{H}^{\circ}\right.$ and $\left.\Delta \mathrm{S}^{\circ}\right)$. The results showed that the isotherm equations that give the best description of copper adsorption were in the following order: Langmuir $>\mathrm{D}-\mathrm{R}>$ Freundlich according to their $\left(\mathrm{R}^{2}\right)$ values. The value of activity energy (E) which ranges from $8.006-15.823 \mathrm{~kJ} \mathrm{~mol}^{-1}$ showed that the copper adsorption is an ionic exchange reaction. The value and sign of $\Delta \mathrm{G}^{\circ}$ of copper adsorption in the studied soils exhibit that it is a spontaneous process and increases with increasing the temperature. For the positive $\Delta \mathrm{H}^{\circ}$ values, $\mathrm{Cu}$ at sorption is considered an endothermic reaction and the positive $\Delta \mathrm{S}^{\circ}$ indicates the degree of randomness between the solid and liquid phases. There is not any clear effect of soil properties. But there is a general effect of whole soil properties on the calculated parameters. The results also showed that the amount of adsorbed $\mathrm{Cu}$ under using 0.01 $\mathrm{M} \mathrm{CaCl}_{2}$ on adsorption sites was lower compared to $0.01 \mathrm{M} \mathrm{NaCl}$.
\end{abstract}

Keywords: Adsorption - Equilibrium - Thermodynamic - Copper. 